\title{
A!
}

This is an electronic reprint of the original article.

This reprint may differ from the original in pagination and typographic detail.

Dasenbrook, D.; Flindt, C.

\section{Dynamical generation and detection of entanglement in neutral leviton pairs}

Published in:

Physical Review B

DOI:

10.1103/PhysRevB.92.161412

Published: 28/10/2015

Document Version

Publisher's PDF, also known as Version of record

Please cite the original version:

Dasenbrook, D., \& Flindt, C. (2015). Dynamical generation and detection of entanglement in neutral leviton pairs. Physical Review B, 92(16), 1-5. [161412]. https://doi.org/10.1103/PhysRevB.92.161412

This material is protected by copyright and other intellectual property rights, and duplication or sale of all or part of any of the repository collections is not permitted, except that material may be duplicated by you for your research use or educational purposes in electronic or print form. You must obtain permission for any other use. Electronic or print copies may not be offered, whether for sale or otherwise to anyone who is not an authorised user. 


\title{
Dynamical generation and detection of entanglement in neutral leviton pairs
}

\author{
David Dasenbrook ${ }^{1}$ and Christian Flindt ${ }^{2}$ \\ ${ }^{1}$ Département de Physique Théorique, Université de Genève, 1211 Genève, Switzerland \\ ${ }^{2}$ Department of Applied Physics, Aalto University, 00076 Aalto, Finland
}

(Received 25 May 2015; revised manuscript received 13 October 2015; published 28 October 2015)

\begin{abstract}
The entanglement of coherently split electron-hole pairs in an electronic conductor is typically not considered accessible due to particle number conservation and fermionic superselection rules. We demonstrate here that current cross-correlation measurements at the outputs of an electronic Mach-Zehnder interferometer can nevertheless provide a robust witness of electron-hole entanglement. Specifically, we consider neutral excitations generated by modulating the transmission of an unbiased quantum point contact periodically in time. For an optimized modulation profile, an entangled state with one positively-charged leviton (a hole) and one negatively-charged leviton (an electron) gets delocalized over the two paths of the interferometer and is detected at the output arms. We evaluate the influence of finite electronic temperatures and dephasing corresponding to recent experiments.
\end{abstract}

Introduction. The development of dynamic single-electron emitters has triggered great interest in gigahertz quantum electronics [1]. Carefully engineered excitations can now be emitted on top of the Fermi sea in an electronic conductor using driven mesoscopic capacitors [2-4] or designed voltage pulses applied to an electrical contact [5,6]. Lorentzianshaped voltage pulses (or a linear drive of a capacitor $[7,8]$ ) excite noiseless quasiparticles known as levitons without accompanying electron-hole pairs [9-12]. These experimental advances open up intriguing avenues for quantum information processing with coherent electrons. One important goal is to generate and detect entanglement of levitons, borrowing ideas and concepts from quantum optics.

Entangled modes with different photon numbers can be generated by sending single photons onto a beam splitter $[13,14]$. Due to particle number superselection rules this type of entanglement has often been considered inaccessible [15]. Further investigations have however clarified that entangled states of different photon numbers provide a resource that is as useful as polarization-entangled photons [16-19]. Advancing similar techniques to entangle states of different electron numbers is clearly desirable, however, the task is challenging. For example, a suitable witness to detect the electronic entanglement must be identified. As such, earlier proposals have instead focused on the orbital entanglement of several electron-hole pairs [20-23] or pairs of electrons [24-26].

In this Rapid Communication we present an experimental recipe for the detection of electron-hole entanglement in an electronic conductor. Specifically, we demonstrate that noise measurements at the outputs of an electronic Mach-Zehnder interferometer, despite the fermionic superselection rules, can provide a robust witness of electron-hole entanglement. An entangled state with one positively-charged leviton and one negatively-charged leviton is produced at a quantum point contact (QPC) and is delocalized across the two arms of the interferometer. Due to particle number conservation the electron-hole entanglement in the state cannot easily be used to violate a Bell inequality [27]. We circumvent this problem by recombining the state at a second QPC. As we show, an entanglement witness can be constructed from crosscorrelation measurements at the output arms. We evaluate the entanglement witness using Floquet theory and find that the electron-hole entanglement can be detected for realistic system parameters, including finite electronic temperatures and dephasing corresponding to recent experiments [28-32].

Mach-Zehnder interferometer. The interferometer consists of a Corbino disk in the quantum Hall regime with electronic motion along edge states from left to right in Fig. 1. The upper and lower arms of the interferometer form a loop that encloses the magnetic flux $\phi$. In addition, electrons above the Fermi level in the upper arm can enter a small cavity which encloses the flux $\phi_{c}$. Two QPCs act as electronic beam splitters. Contrary to recent experiments, all contacts are grounded. Instead, we modulate the transmission probability of the first QPC periodically in time in such a way that clean electron-hole excitations are generated out of the otherwise undisturbed Fermi sea at the location of the QPC. Each electron-hole pair delocalizes across the arms of the interferometer, leading to a superposition of a negatively-charged leviton being in the upper arm and a positively-charged leviton in the lower arm and vice versa. As we go on to show, the resulting electron-hole entanglement can be detected by measuring the cross correlations of the currents in the output arms after the second QPC.

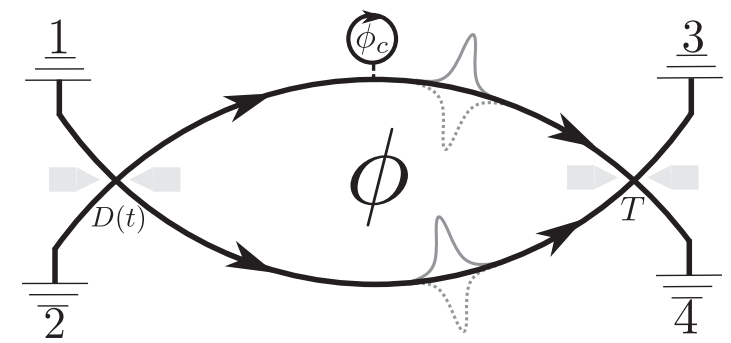

FIG. 1. Dynamic Mach-Zehnder interferometer. Entangled states of neutral leviton pairs are generated by modulating the transmission $D(t)=|d(t)|^{2}$ of the first QPC periodically in time. The levitons travel along edge states to the second QPC with transmission $T$, and the entanglement is detected by measuring the current cross correlations at the output arms. The interferometer encloses the magnetic flux $\phi$. Negatively-charged levitons in the upper part can enter a small cavity with magnetic flux $\phi_{c}$ and pick up an additional phase. 
Dynamic entanglement generation. We start with the generation of clean electron-hole pairs at a QPC. This problem is closely related to the creation of levitons by applying Lorentzian-shaped voltage pulses to a contact [9-12]. As predicted by Levitov and co-workers and recently realized experimentally $[5,6]$, pulses of the form

$$
V(t)=-\frac{\hbar}{e} \sum_{n=-\infty}^{\infty} \frac{2 \eta}{(t+n \mathcal{T})^{2}+\eta^{2}}
$$

lead to the emission of levitons from the contact on top of the otherwise undisturbed Fermi sea (with a holelike leviton going into the contact). The width of the pulses is $\eta$ and $\mathcal{T}$ the period of the driving. Levitons are created as each electron leaving the contact picks up the phase factor $e^{i \varphi(t)}$ with the phase given as

$$
\varphi(t)=-\frac{e}{\hbar} \int_{0}^{t} \mathrm{~d} t^{\prime} V\left(t^{\prime}\right)
$$

The phase changes sign $\varphi(t) \rightarrow-\varphi(t)$ upon inverting the voltage $V(t) \rightarrow-V(t)$, leading to the emission of a holelike leviton from the contact.

Remarkably, a similar strategy can be used to generate superpositions of electronlike and holelike levitons by modulating the transmission of a QPC periodically in time [33,34]. To see this, we consider the time-dependent scattering matrix of the first QPC in Fig. 1,

$$
S(t)=\left[\begin{array}{cc}
r(t) & d(t) \\
-d(t) & r(t)
\end{array}\right],
$$

where the reflection and transmission amplitudes, chosen to be real below, fulfill $|r(t)|^{2}+|d(t)|^{2}=1$. Switching to the eigenbasis of $S(t)$, particles in the two incoming eigenchannels will be completely reflected with the reflection amplitudes $r(t) \pm i d(t)$ given by the eigenvalues of $S(t)$. We now choose the transmission and reflection as

$$
\begin{aligned}
& d(t)=\sin \varphi(t), \\
& r(t)=\cos \varphi(t),
\end{aligned}
$$

with $\varphi(t)$ given by Eq. (2). The reflection amplitudes in the eigenbasis then become $r(t) \pm i d(t)=e^{ \pm i \varphi(t)}$, implying that an electronlike leviton is reflected in one eigenchannel and a holelike leviton in the other. Returning to the physical channels of the QPC, the outgoing state after the small cavity in Fig. 1 becomes

$$
|\Psi\rangle=\frac{1}{2}\left(e^{i \vartheta} \hat{b}_{u-}^{\dagger} \hat{b}_{u+}^{\dagger}-\hat{b}_{l-}^{\dagger} \hat{b}_{l+}^{\dagger}+i\left\{e^{i \vartheta} \hat{b}_{u-}^{\dagger} \hat{b}_{l+}^{\dagger}+\hat{b}_{u+}^{\dagger} \hat{b}_{l-}^{\dagger}\right\}\right)|0\rangle,
$$

where $|0\rangle$ is the filled Fermi sea at zero temperature and the Fermi energy is zero. The operators $\hat{b}_{i-}^{\dagger}=\sum_{E>0} e^{-\eta E} \hat{b}_{i}^{\dagger}(E)$ and $\hat{b}_{i+}^{\dagger}=\sum_{E<0} e^{\eta E} \hat{b}_{i}(E)$ create electronlike and holelike levitons in the upper $(i=u)$ or lower $(i=l)$ arms of the interferometer, and $\hat{b}_{i}^{\dagger}(E)$ creates electrons at energy $E$ in either of the two arms. We assume for now that the effect of the small cavity can be encoded in a tunable phase $\vartheta=\vartheta\left(\phi_{c}\right)$ picked up by electronlike levitons in the upper arm. Below, we return to a more detailed description of the cavity [see Eq. (11)]. As Eq. (5) cannot be written as a product of $b_{u}^{\dagger}$ and $b_{l}^{\dagger}$ operators, the state is entangled. Furthermore, the projection of the state on the subspace with one particle per arm (in curly brackets) is maximally entangled in the electron-hole degree of freedom. This is the entanglement we wish to detect. We note that the state in Eq. (5) can also be generated by emitting levitons from different inputs onto the QPC tuned to half transmission using quantum capacitors with a linear drive $[7,8]$.

Entanglement witness. It is difficult to formulate a Bell inequality for the entanglement between modes of different particle numbers. (It would require measurements in a basis of nondefinite particle number, and for electrons a superconductor, for instance, would be needed [35]). Furthermore, the violation of Bell inequalities often relies on very high visibilities and is therefore currently out of reach for mesoscopic conductors. To circumvent these problems, we instead construct an entanglement witness based on current cross-correlation measurements at the output arms after the second QPC, similar to what has been considered in the context of spin entanglement [36,37]. To develop our witness, we consider a general two-leviton state incident on the second QPC

$$
|\Upsilon\rangle=\sum_{\substack{\alpha, \beta= \pm i, j=u, l}} \Upsilon_{\alpha \beta}^{i j} \hat{b}_{i \alpha}^{\dagger} \hat{b}_{j \beta}^{\dagger}|0\rangle
$$

with the normalization condition $\sum_{\alpha \beta i j}\left|\Upsilon_{\alpha \beta}^{i j}\right|^{2}=1$. Equation (5) is a particular example of such a state.

If the projection on the single-particle sector is separable, the matrix $\Upsilon^{u l}$ has rank one [38]. Calculating the cross correlator $S_{34}(\phi)=\left\langle\hat{I}_{3} \hat{I}_{4}\right\rangle$ measured after the second QPC, we can then show that the function [39]

$$
f(\phi) \equiv S_{34}(\phi)-S_{0}(1-2 T R)
$$

is always zero or negative. Here, $S_{0}=\sum_{\alpha \beta} \alpha \beta\left|\Upsilon_{\alpha \beta}^{u l}\right|^{2}$ is the noise at zero transmission and $\phi=2 \pi \Phi / \Phi_{0}$ is the Aharonov-Bohm phase with $\Phi$ being the magnetic flux enclosed by the interferometer and $\Phi_{0}=h / e$ the magnetic flux quantum. Moreover, for a general separable density matrix $\hat{\rho}=\sum_{n} p_{n}\left|\Upsilon_{n}\right\rangle\left\langle\Upsilon_{n}\right|$ [40] with each $\left|\Upsilon_{n}\right\rangle$ of the form (6) and separable, the noise is the average noise of each separable state weighted by the probabilities $p_{n}$. Therefore, the condition $f(\phi)>0$ provides a witness of electron-hole entanglement also at finite temperatures. The witness is not optimal, since negative two-particle contributions to the noise [terms with $i=j=u$ or $i=j=l$ in Eq. (6)] can make it harder to detect the entanglement, even if it is maximal [40]. Importantly, our witness relies on reconnecting the two arms at the second QPC, making the measurement nonlocal. This is the key ingredient that allows us to circumvent the superselection rules for particle number.

Evaluating the witness for the state in Eq. (5), we first find a pure interference current determined by the enclosed fluxes, $\left\langle\hat{I}_{3}\right\rangle=-\left\langle\hat{I}_{4}\right\rangle=e(\Omega / \pi) \sqrt{T R} \cos \left[\vartheta\left(\phi_{c}\right) / 2\right] \sin [\phi+$ $\vartheta\left(\phi_{c}\right) / 2$ ], where $\Omega=2 \pi / \mathcal{T}$ is the frequency of the driving. For the current cross correlator, we find

$$
S_{34}=-\frac{e^{2} \Omega}{4 \pi}[1-2 T R\{1-\cos (2 \phi)\}]+\left\langle\hat{I}_{3}\right\rangle\left\langle\hat{I}_{4}\right\rangle .
$$

Both the current and the noise are independent of the pulse width $\eta$, which determines the spatial extent of the levitons. 

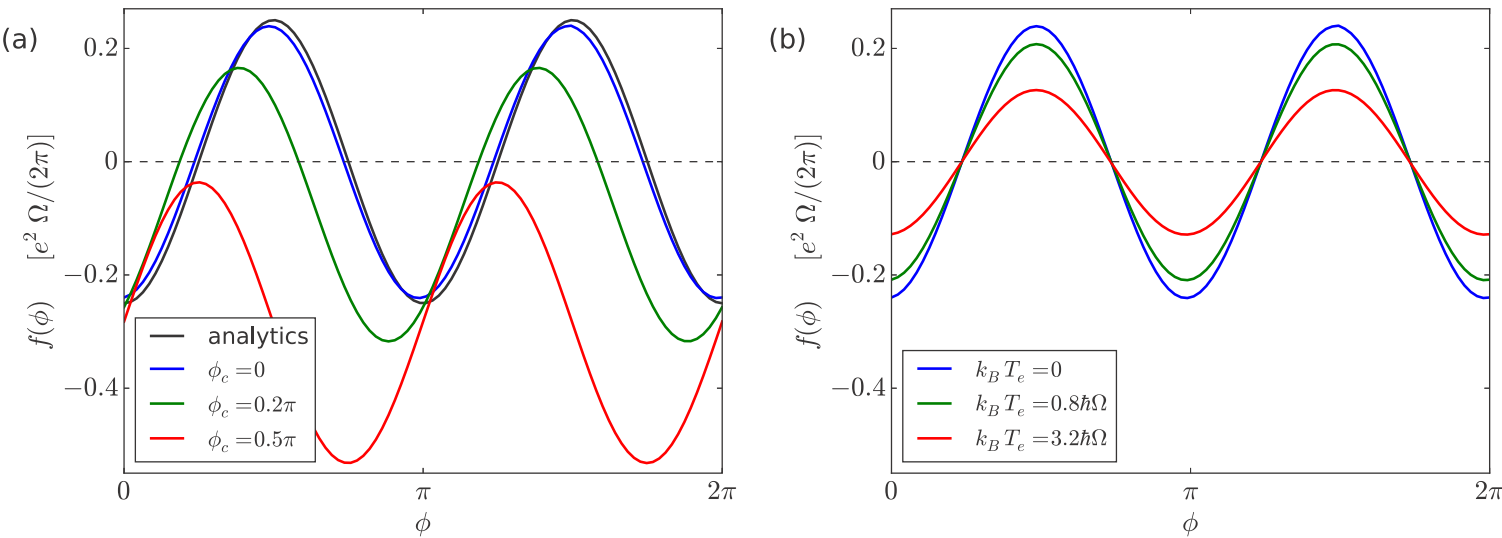

FIG. 2. (Color online) Entanglement witness. (a) The witness $f(\phi)$ as a function of the Aharonov-Bohm phase $\phi$ with different values of the flux $\phi_{c}$ enclosed by the small cavity. The electronic temperature is zero. Positive values of $f$ (above the dashed line) signal electron-hole entanglement. With $\phi_{c}=0$, the Floquet calculation is close to the analytic result $-\cos (2 \phi) / 4$ (black line) corresponding to the maximally entangled state in Eq. (5). The parameters of the cavity are $\tau=\mathcal{T} / 50$ and $\mathcal{B}=5 \mathcal{T} / \hbar$. (b) The witness $f(\phi)$ as a function of $\phi$ with $\phi_{c}=0$ and different electronic temperatures $T_{e}$. An increased temperature merely decreases the amplitude of the oscillations so that the entanglement is still detectable at finite temperatures.

Now, tuning the phase $\vartheta\left(\phi_{c}\right)$ to $\pi$ and choosing $T=R=1 / 2$, the average currents vanish and the witness becomes $f(\phi)=$ $-e^{2} \Omega /(8 \pi) \cos (2 \phi)$, which clearly can be positive signaling electron-hole entanglement.

Floquet scattering theory. The noise in Eq. (8) corresponds to the ideal case of the entangled state in Eq. (5). We now proceed with a full Floquet calculation [41,42] of the entanglement witness for the interferometer in Fig. 1, including finite electronic temperatures and a detailed description of the small cavity. In this case, the outgoing state is not known and we need to evaluate the witness to detect the entanglement. As we will see, electron-hole entanglement is detectable under realistic experimental conditions.

The current operator in contact $i$ can be written as $\hat{I}_{i}=$ $\frac{e}{h} \int_{-\infty}^{\infty} \mathrm{d} E\left(\hat{c}_{i}^{\dagger}(E) \hat{c}_{i}(E)-\hat{a}_{i}^{\dagger}(E) \hat{a}_{i}(E)\right)$ in terms of the operators for particles at energy $E$ incoming from and outgoing to reservoir $i$, respectively. The operators for outgoing particles can be expressed as

$$
\hat{c}_{i}(E)=\sum_{j} \sum_{n=-\infty}^{\infty} \mathcal{S}_{i j}\left(E, E_{n}\right) \hat{a}_{j}\left(E_{n}\right),
$$

where $\mathcal{S}$ is the Floquet scattering matrix and $\hat{a}_{j}(E)$ are operators for incoming particles from reservoir $j$.

The Floquet amplitudes for incoming particles at energy $E$ to scatter into the outgoing reservoirs with energy $E_{n}=$ $E+n \hbar \Omega$, having absorbed $(n>0)$ or emitted $(n<0)|n|$ energy quanta of size $\hbar \Omega$, read

$$
\begin{aligned}
& \mathcal{S}_{31}\left(E_{n}, E\right)=-\sqrt{T} S_{F}^{d}(n)+\sqrt{R} e^{i \phi} S_{c}\left(E_{n}\right) S_{F}^{r}(n), \\
& \mathcal{S}_{32}\left(E_{n}, E\right)=\sqrt{T} S_{F}^{r}(n)-\sqrt{R} e^{i \phi} S_{c}\left(E_{n}\right) S_{F}^{d}(n), \\
& \mathcal{S}_{41}\left(E_{n}, E\right)=\sqrt{R} S_{F}^{d}(n)+\sqrt{T} e^{i \phi} S_{c}\left(E_{n}\right) S_{F}^{r}(n), \\
& \mathcal{S}_{42}\left(E_{n}, E\right)=-\sqrt{R} S_{F}^{r}(n)-\sqrt{T} e^{i \phi} S_{c}\left(E_{n}\right) S_{F}^{d}(n) .
\end{aligned}
$$

Here, the Floquet amplitudes of the first QPC, $S_{F}^{s}(n)=$ $\int_{0}^{\mathcal{T}} \mathrm{d} t s(t) e^{i n \Omega t} / \mathcal{T}$, with $s=d, r$ given in Eq. (4), are $S_{F}^{d}(n \neq$ $0)=-\sinh (2 \pi \eta) e^{-2 \pi|n| \eta}, S_{F}^{d}(n=0)=e^{-2 \pi \eta}$ and $S_{F}^{r}(n)=$ $\operatorname{sgn}(n) \sinh (2 \pi \eta) e^{-2 \pi|n| \eta}$. In addition, the scattering matrix of the small cavity reads

$$
S_{c}(E)=r_{c}(E)+t_{c}^{2}(E) \frac{e^{i\left(\phi_{c}+E \tau / \hbar+\pi\right)}}{1+r_{c}(E) e^{i\left(\phi_{c}+E \tau / \hbar+\pi\right)}},
$$

where $\tau$ is the time it takes to complete one loop inside the cavity and $t_{c}(E)=1 /[\exp (-\mathcal{B} E)+1]$ is the transmission amplitude into the cavity with the cutoff $\mathcal{B}$ being tunable by a magnetic field $[43,44]$. The reflection amplitude is $r_{c}(E)=\sqrt{1-t_{c}^{2}(E)}$. With a sharp cutoff $\mathcal{B} \gg \mathcal{T} / h$ and a short loop time $\tau \ll \mathcal{T}$, we recover the state in Eq. (5) with $\vartheta\left(\phi_{c}\right) \simeq \phi_{c}+\pi$.

Figure 2 shows the entanglement witness calculated using Floquet scattering theory $[41,42]$. We vary the AharonovBohm phase $\phi$ and show in panel (a) results for different values of the flux $\phi_{c}$ enclosed by the cavity. The electronic temperature is zero. The entanglement cannot be detected in all situations. However, by tuning $\phi_{c}$ we come close to the analytic result (black line) corresponding to the maximally entangled state in Eq. (5). The system then maximally violates the inequality $f(\phi) \leqslant 0$ in the sense that the witness has the same weight above and below the $f=0$ line as a function of $\phi$. Under this condition, the witness is thus expected to be very robust against a decreased visibility, in contrast to entanglement detection based on Bell inequalities [26].

In panel (b) we fix the optimal value of $\phi_{c}=0$ and consider the effect of a finite electronic temperature. With increasing temperature, the amplitude of the oscillations decreases and the entanglement gets harder to detect. Still, even with temperatures that are higher than the driving frequency, the witness can become positive and entanglement can be detected. Since there is no direct scattering path between the two output reservoirs, thermal noise is not visible in the witness [45]. The results in Fig. 2 are promising for the detection of electron-hole entanglement in driven mesoscopic conductors. 


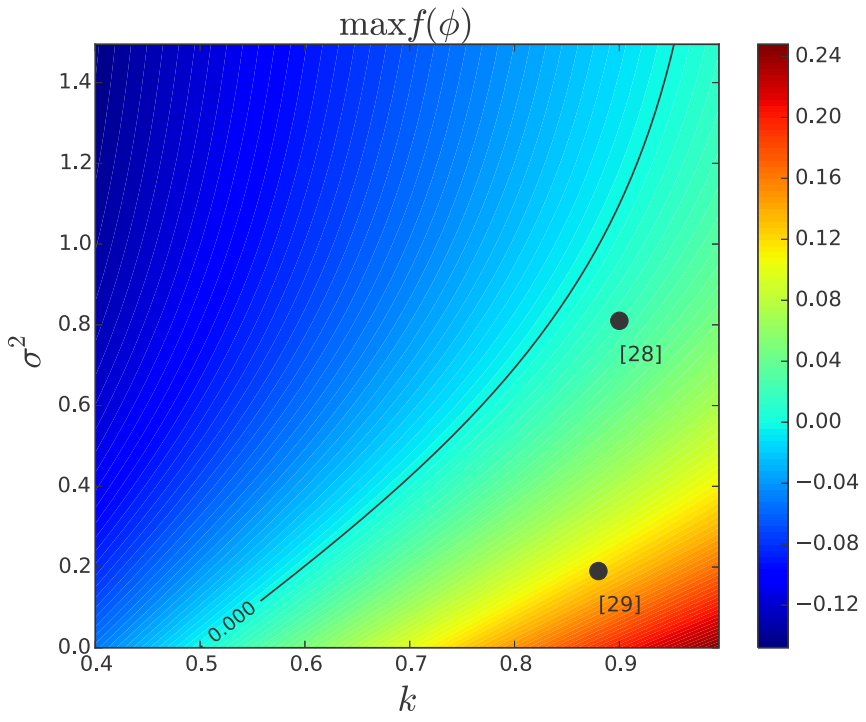

FIG. 3. (Color online) Influence of dephasing. The maximum value of the entanglement witness $f(\phi)$ as a function of the decoherence parameter $k$ and the variance of the phase $\sigma^{2}$. The contour line separates the region of detectable electron-hole entanglement, where the witness is positive, from the region where the entanglement cannot be detected. The black dots mark the experimental parameters from Refs. [28,29], which lie in the region of detectable entanglement.

Dephasing mechanisms. Finally, to estimate the influence of dephasing and phase averaging, we return to the analytic result for the noise in Eq. (8). Focusing on the optimal value $\vartheta=\pi$, the noise can be written as $S_{34}=-e^{2} \Omega /(4 \pi)[1-2 k T R\{1-$ $\left.\left.\exp \left(-2 \sigma^{2}\right) \cos (2 \phi)\right\}\right]$ in terms of the phenomenological parameters $k$ [28] and $\sigma^{2}$ [30] which describe the coherence of the wave function across the interferometer $(k=1$ meaning full coherence and $k=0$ no coherence, e.g., as a result of a finite electronic temperature or interactions) and the variance of the total Aharonov-Bohm phase leading to phase averaging. In Fig. 3 we show the maximal value of the witness $f(\phi)$ as a function of $k$ and $\sigma^{2}$. We find that the witness is robust against moderate dephasing mechanisms and that entanglement is detectable for parameters corresponding to the experiments reported in Refs. [28,29]. This is in contrast to the detection of orbital entanglement based on a Bell inequality [26].

Conclusions. We have proposed and analyzed a dynamical scheme to generate and detect entanglement in the electronhole degree of freedom of leviton pairs. Measuring the cross correlations at the output arms of a mesoscopic MachZehnder interferometer, entanglement can be detected despite superselection rules. The entanglement witness is robust against moderate dephasing mechanisms, and entanglement can be detected using current technologies. Future work may investigate the entanglement entropy generated in this scheme [46-49].

Acknowledgments. We thank J. Bowles, J. B. Brask, N. Brunner, D. C. Glattli, P. P. Hofer, J. R. Ott, P. Roulleau, and K. H. Thomas for helpful discussions. C.F. is affiliated with Centre for Quantum Engineering at Aalto University. D.D. gratefully acknowledges the hospitality of Aalto University. This work was supported by the Swiss NSF and Academy of Finland.
[1] E. Bocquillon, V. Freulon, F. D. Parmentier, J.-M. Berroir, B. Placais, C. Wahl, J. Rech, T. Jonckheere, T. Martin, C. Grenier, D. Ferraro, P. Degiovanni, and G. Fève, Ann. Phys. 526, 1 (2014).

[2] J. Gabelli, G. Fève, J.-M. Berroir, B. Plaçais, A. Cavanna, B. Etienne, Y. Jin, and D. C. Glattli, Science 313, 499 (2006).

[3] G. Fève, A. Mahè, J.-M. Berroir, T. Kontos, B. Plaçais, D. C. Glattli, A. Cavanna, B. Etienne, and Y. Jin, Science 316, 1169 (2007).

[4] E. Bocquillon, V. Freulon, J.-M. Berroir, P. Degiovanni, B. Plaçais, A. Cavanna, Y. Jin, and G. Fève, Science 339, 1054 (2013).

[5] J. Dubois, T. Jullien, F. Portier, P. Roche, A. Cavanna, Y. Jin, W. Wegscheider, P. Roulleau, and D. C. Glattli, Nature (London) 502, 659 (2013).

[6] T. Jullien, P. Roulleau, B. Roche, A. Cavanna, Y. Jin, and D. C. Glattli, Nature (London) 514, 603 (2014).

[7] M. Büttiker, H. Thomas, and A. Prêtre, Phys. Lett. A 180, 364 (1993).

[8] J. Keeling, A. V. Shytov, and L. S. Levitov, Phys. Rev. Lett. 101, 196404 (2008).

[9] D. A. Ivanov, H. W. Lee, and L. S. Levitov, Phys. Rev. B 56, 6839 (1997).

[10] L. S. Levitov, H. Lee, and G. B. Lesovik, J. Math. Phys. 37, 4845 (1996).
[11] J. Keeling, I. Klich, and L. S. Levitov, Phys. Rev. Lett. 97, 116403 (2006).

[12] J. Dubois, T. Jullien, C. Grenier, P. Degiovanni, P. Roulleau, and D. C. Glattli, Phys. Rev. B 88, 085301 (2013).

[13] G. Björk, P. Jonsson, and L. L. Sánchez-Soto, Phys. Rev. A 64, 042106 (2001).

[14] S. J. van Enk, Phys. Rev. A 72, 064306 (2005).

[15] H. M. Wiseman and J. A. Vaccaro, Phys. Rev. Lett. 91, 097902 (2003).

[16] E. Lombardi, F. Sciarrino, S. Popescu, and F. De Martini, Phys. Rev. Lett. 88, 070402 (2002).

[17] S. D. Bartlett, T. Rudolph, and R. W. Spekkens, Rev. Mod. Phys. 79, 555 (2007).

[18] D. Salart, O. Landry, N. Sangouard, N. Gisin, H. Herrmann, B. Sanguinetti, C. Simon, W. Sohler, R. T. Thew, A. Thomas, and H. Zbinden, Phys. Rev. Lett. 104, 180504 (2010).

[19] S. Takeda, M. Fuwa, P. van Loock, and A. Furusawa, Phys. Rev. Lett. 114, 100501 (2015).

[20] C. W. J. Beenakker, C. Emary, M. Kindermann, and J. L. van Velsen, Phys. Rev. Lett. 91, 147901 (2003).

[21] P. Samuelsson, E. V. Sukhorukov, and M. Büttiker, Phys. Rev. Lett. 92, 026805 (2004).

[22] P. Samuelsson and M. Büttiker, Phys. Rev. B 71, 245317 (2005).

[23] Y. Sherkunov, N. d'Ambrumenil, P. Samuelsson, and M. Büttiker, Phys. Rev. B 85, 081108 (2012). 
[24] P. Samuelsson, E. V. Sukhorukov, and M. Büttiker, Phys. Rev. Lett. 91, 157002 (2003).

[25] H.-S. Sim and E. V. Sukhorukov, Phys. Rev. Lett. 96, 020407 (2006).

[26] P. Samuelsson, I. Neder, and M. Büttiker, Phys. Rev. Lett. 102, 106804 (2009).

[27] N. Brunner, D. Cavalcanti, S. Pironio, V. Scarani, and S. Wehner, Rev. Mod. Phys. 86, 419 (2014).

[28] Y. Ji, Y. Chung, D. Sprinzak, M. Heiblum, D. Mahalu, and H. Shtrikman, Nature (London) 422, 415 (2003).

[29] I. Neder, N. Ofek, Y. Chung, M. Heiblum, D. Mahalu, and V. Umansky, Nature (London) 448, 333 (2007).

[30] P. Roulleau, F. Portier, D. C. Glattli, P. Roche, A. Cavanna, G. Faini, U. Gennser, and D. Mailly, Phys. Rev. B 76, 161309 (2007).

[31] L. V. Litvin, A. Helzel, H.-P. Tranitz, W. Wegscheider, and C. Strunk, Phys. Rev. B 78, 075303 (2008).

[32] P.-A. Huynh, F. Portier, H. le Sueur, G. Faini, U. Gennser, D. Mailly, F. Pierre, W. Wegscheider, and P. Roche, Phys. Rev. Lett. 108, 256802 (2012).

[33] Y. Sherkunov, J. Zhang, N. d'Ambrumenil, and B. Muzykantskii, Phys. Rev. B 80, 041313(R) (2009).

[34] J. Zhang, Y. Sherkunov, N. d'Ambrumenil, and B. Muzykantskii, Phys. Rev. B 80, 245308 (2009).
[35] C. W. J. Beenakker, Phys. Rev. Lett. 112, 070604 (2014).

[36] G. Burkard and D. Loss, Phys. Rev. Lett. 91, 087903 (2003).

[37] V. Giovannetti, D. Frustaglia, F. Taddei, and R. Fazio, Phys. Rev. B 74, 115315 (2006).

[38] L. Amico, R. Fazio, A. Osterloh, and V. Vedral, Rev. Mod. Phys. 80, 517 (2008).

[39] See Supplemental Material at http://link.aps.org/supplemental/ 10.1103/PhysRevB.92.161412 for a derivation of the entanglement witness Eq. (7) in the manuscript.

[40] R. Horodecki, P. Horodecki, M. Horodecki, and K. Horodecki, Rev. Mod. Phys. 81, 865 (2009).

[41] M. Moskalets and M. Büttiker, Phys. Rev. B 66, 205320 (2002).

[42] M. Moskalets, Scattering Matrix Approach to Non-Stationary Quantum Transport (Imperial College Press, London, 2011).

[43] H. A. Fertig and B. I. Halperin, Phys. Rev. B 36, 7969 (1987).

[44] M. Büttiker, Phys. Rev. B 41, 7906 (1990).

[45] P. P. Hofer and C. Flindt, Phys. Rev. B 90, 235416 (2014).

[46] I. Klich and L. S. Levitov, Phys. Rev. Lett. 102, 100502 (2009).

[47] H. F. Song, C. Flindt, S. Rachel, I. Klich, and K. Le Hur, Phys. Rev. B 83, 161408 (2011).

[48] H. F. Song, S. Rachel, C. Flindt, I. Klich, N. Laflorencie, and K. Le Hur, Phys. Rev. B 85, 035409 (2012).

[49] K. H. Thomas and C. Flindt, Phys. Rev. B 91, 125406 (2015). 